\title{
Antihyperglycemic and Antihyperlipidemic Effect of Persea americana in High Fat Diet and Low Dose Streptozotocin Induced T2DM Male Albino Wistar Rats
}

\section{Ture Girma}

Dilla College: Dilla University

\section{Solomon Genet}

Addis Ababa University

\section{Teka Obsa Obsa Feyisa ( $\square$ teka.feyisa@haramaya.edu.et)}

Haramaya University College of Health and Medical Sciences https://orcid.org/0000-0002-8424-3004

\section{Abdissa Tufa}

Addis Ababa University

\section{Research}

Keywords: Antihyperglycemic effect, T2DM, Persea americana, High fat diet, STZ, hyperglycemia, dyslipidemia, Albino Wistar rats

Posted Date: February 9th, 2021

DOI: https://doi.org/10.21203/rs.3.rs-198182/v1

License: (c) (i) This work is licensed under a Creative Commons Attribution 4.0 International License. Read Full License 


\section{Abstract}

Background: Type II diabetes is a major health problem worldwide, and is increasing in an alarming rate globally and in Ethiopia due to change in dietary habits and sedentary life style. Even though there is no effective cure for diabetes, early control of blood glucose significantly reduces the risk of diabetic complications. Different types of ingredients present in medicinal plants that act on a variety of targets by various modes and mechanisms are used to treat diabetes with minimum cost and side effect. Therefore, the objective of the present study was to investigate the antidiabetic effect of Persea americana mill fruit juice in high fat diet (HFD) and low dose Streptozotocin (STZ) induced type 2 diabetic (T2DM) male albino Wistar rats.

Methods: Thirty six male albino Wistar rats weighing form 150-200g were divided in into six different groups: group I (normal control); Group II (diabetic control); Group III (metformin control) and Group IV - Group VI (treatment groups). Group I was fed on standard pellet and group II - group VI were fed on HFD for 4 weeks to induce pre-diabetes and insulin resistance followed by low dose STZ injection to induce T2DM. The treatment groups (group IV, V and VI) were given $632 \mathrm{mg} / \mathrm{Kg}, 1264 \mathrm{mg} / \mathrm{Kg}$ and $1896 \mathrm{mg} / \mathrm{Kg} /$ day of Persea americana fruit juice for six weeks, respectively to compare with normal, diabetic and $7 \mathrm{mg} / \mathrm{Kg}$ metformin treated groups. After forty-five days of treatment, the rats were fasted overnight (12 to 14 hours), anaesthetized and blood sample was collected by cardiac puncture for biochemical tests (fasting blood glucose (FBG), lipid profile, total protein and creatinine). The results were analyzed using SPSS version 22.0. One way ANOVA followed by Post hoc Tukey's multiple comparisons were done to compare the mean differences among the experimental groups, and $p$-values < 0.05 were considered statistically significant.

Results: In high dose (1896 mg/Kg/day) Persea americana mill fruit juice treated group, food consumption, body weight, FBG, and LDL-C were significantly reduced and HDL-C was significantly increased $(p<0.005)$ compared with diabetic control group. Moderate dose $(1264 \mathrm{mg} / \mathrm{Kg} /$ day $)$ treated group showed a decrease in FBG on $6^{\text {th }}$ week and improve HDL-C levels. Treating the rats with Persea americana fruit juice changed TG, total protein and creatinine levels although not significant. Oral antidiabetes drug (metformin) significantly reduced pellet consumption, body weight, FBG and lipid profile.

Conclusion: Overall, Persea americana mill fruit juice showed antihyperglycemic and antihyperlipidemic effect particularly through reduction of fasting blood glucose, LDL-C and increasing HDL-C in T2DM induced rats, thus it can be helpful in reducing the risk of diabetic complications.

\section{Background}

Diabetes mellitus (DM) is a metabolic disorder, which either results from deficiency in insulin production by the pancreas or inability of the insulin produced to bind effectively to its receptor on the cell surface. Either of these conditions leads to accumulation of glucose in the blood and results in chronic hyperglycaemia $(1,2)$. Untreated chronic hyperglycaemia can in turn lead to long-term complications, including micro-vascular and macro-vascular problems that cause disturbances of carbohydrate, fat and protein metabolism, and it covers a wide range of heterogeneous diseases (3). World health organization (WHO) estimated that globally, 425 million adults aged over 18 years were living with diabetes in 2017 and this will increase to 629 million by 2045 (4). The diabetes prevalence in sub-Saharan Africa, which was 12.1 million in 2016 is expected to rise to 23.9 million by 2030 (5). 
Diabetes mellitus could be categorized into several groups: type 1 diabetes mellitus (T1DM), type 2 diabetes mellitus (T2DM), gestational diabetes, and other specific types, but the vast majority cases of diabetes fall into T1DM and T2DM broad categories (6). Type 1 diabetes is arisen from absolute deficiency of insulin secretion while the more prevalent category, T2DM, is due to combination of resistance to insulin action and an inadequate compensatory insulin secretory response (7). Both T1DM and T2DM are characterized by hyperglycaemia, excessive urine production, compensatory thirst, increased fluid intake, blurred vision, unexplained weight loss, lethargy, and changes in energy metabolism (8).

Type 2 DM is the most common type of diabetes and accounts for $90-95 \%$ of all diabetic cases, and mostly prevalent in people older than 45 years who are overweight (9). It is characterized by a significant insulin production ranging from less than normal to above normal, but the body is unable to utilize it efficiently $(9,10)$. The change in dietary habits and a sedentary lifestyle are the two main causes responsible for the development of T2DM when insulin resistance is established as a precondition $(1,12)$.

Yet, there is no effective cure for diabetes, and the oral antihperglycemic drugs and insulin currently used in managing the disease are associated with several undesirable side effects. The use of oral antidiabetic drugs is limited due to their adverse side effects such as the haematological, cutaneous and gastrointestinal reactions, hypoglycaemic coma and impairment of liver and kidney functions. In addition, they are not suitable to use during pregnancy $(2,8)$. Moreover, providing a modern medical healthcare across the world is still a far-off goal due to economic constraints (13).

Apart from the currently available therapeutic options, many herbal medicines have been recommended for the treatment of diabetes. A variety of ingredients present in medicinal plants are thought to act on a variety of diabetic targets by various modes and mechanisms (14). Many important drugs used in medicine today are directly or indirectly derived from plants due to its bioactive constituents such as; alkaloids, steroids, tannins and some plant phytochemicals, including cafestol, flavonoids and carotenoids (15).

Persea americana is the tree originated from Mexico and nowadays cultivated in different parts of the world, including Ethiopia $(8,13)$. The phytochemical constituents of Persea americana have been studied and analysis had shown the presence of polyphenols that has one to two times more protein than any other fruit, contains low amount of simple sugar and appreciable amount of dietary fiber, high in manganese, phosphorous, iron and potassium, but low in sodium, vitamin E, vitamin C, carotene, thiamin, riboflavin, nicotinic acid and folate (16). Persia ameriana fruit pulp has a various beneficial health effects such as anti-obesity, hepatoprotective, antiosteoarthritis, anti-carcinogenic effects, suppressing liver injury, wound healing activity and chemo-protective activities (17-21). It has also been reported to exhibit several pharmacological effects in ethnomedicine, including treatment for diarrhoea, intestinal parasites, skin and seed oil for weight loss $(22,23)$. Thus, the present study was aimed to investigate the antidiabetic effects of Persia ameriana fruit pulp juice in high fat diet and low dose SZT induced diabetic male albino Wistar rats.

\section{Methods And Materials}

\section{Study Design and Period}

An experimental study was conducted at animal laboratory of Biochemistry Department, College of Health Sciences, Addis Ababa University, Etiopia from December 2018 to May 2019 to investigate the antidiabetic effect 
of Persea americana fruit pulp juice in male albino Wistar rats.

\section{Ethical Consideration}

The study was conducted after the proposal was evaluated and approved by Department of Biochemistry Research and Ethical Review Committee (DRERC) by protocol no. M.Sc.13/17. All experimental activities conducted in the laboratory was in accordance to ethical declaration of national and international standards for experimental animals, which protect the right of experimental animal and minimize suffer, hunger, pain, thirst, injury, discomfort and fear to the best minimal level.

\section{Sample Size Determination}

An appropriate sample size determination is important since too small size misses the real effect in an experiment while a sample size larger than necessary will lead to wasting resources and ethical issues on sacrificed animals (24). Number of animals was calculated according to Federer rule:(k-1) (n-1) $\geq 15$ where $k$, is the number of groups and $n$, number of subjects per group; and drop-out size (do) was estimated 10\%, the minimal size of sample was determined as: $n d o=5 /(1-d o)^{2}=5 /(1-0.1)^{2}=5 / 0.81=6.2 \approx 6$ where ndo is minimal sample size (25). The size of sample in each group was at 6 rats. The study was conducted on six groups, so the total sample size was 6 groups $\times 6$ rats $=36$ rats.

\section{Experimental Animals and Study Protocol}

Male albino Wistar rats (4-5 weeks) weighing 150-200 g were obtained from Department of Pharmacology, College of Health Sciences, Addis Ababa University. The animals were housed in polypropylene plastic cages and maintained under standard laboratory conditions of room/optimum temperature and 12 hour light/dark cycle at Biochemistry animal laboratory and fed on a standard commercial rat pellet and water ad libitum. At 9 - 10 weeks of age, the rats were randomly assigned into six groups. Throughout the experiment, each rat in the assigned group was identified by giving a specific number on its tail by permanent marker. The allocation and treatment of the rats were as follows:

Group I: Normal control rats injected citrate buffer and administered $3.5 \mathrm{ml} / \mathrm{Kg}$ of distilled water orally

Group II: HFD and STZ induced diabetic rats that served as diabetic control and were given distilled water daily.

Group III: HFD and STZ induced diabetic rats treated with $7 \mathrm{mg} / \mathrm{Kg}$ of metformin orally

Group IV: HFD and STZ induced diabetic rats treated with $632 \mathrm{mg} / \mathrm{Kg}$ of Persea americana fruit juice.

Group V: HFD and STZ induced diabetic rats treated with $1264 \mathrm{mg} / \mathrm{Kg}$ of

Persea Americana fruit juice.

Group Vl: HFD and STZ induced diabetic rats treated with 1896 mg/Kg-BW/day of Persea Americana fruit juice.

\section{High Fat Diet (HFD) Preparation}

Many experiments have used rats fed with commercial lard (animal fat) as an obesity model (26). However, since commercial lard is not available in Ethiopia, the HFD used in this experiment was prepared from locally available 
bovine fat. To prepare the HFD, $40 \%$ purified fat was mixed with $60 \%$ of pellet $(40 \% / 60 \% \mathrm{w} / \mathrm{w})$. Since the standard pellet powder consists of $20 \%$ fat, $60 \%$ carbohydrate and $20 \%$ protein (27), the final percentage of fat is $52 \%$ (i.e., $52 \% / 100 \% \mathrm{~W} / \mathrm{W})$. Hence, the term, 'high fat diet' used in this study refers to a diet containing $52 \%$ fat, $36 \%$ carbohydrate and $12 \%$ of protein.

\section{Induction of Type 2 Diabetes Mellitus}

There are different methods to induce diabetes mellitus in animal model (28). In our study, high fat diet (HFD) and low dose streptozotocin (STZ) induced diabetic rat model was used. This model involves a combination of a high fat diet to bring about hyperinsulinemia, insulin resistance and glucose intolerance followed by treatment with the $\beta$ cell toxin STZ, which results in a severe reduction in functional $\beta$ cell mass. These two stressors were designed to induce the features of human T2DM pathology to the rats within a shorter period of time scale.

\section{Streptozotocin Injection}

After 4 weeks of dietary manipulation, the rats were fasted overnight and injected intraperitoneally with freshly prepared STZ at a concentration of $35 \mathrm{mg} / \mathrm{Kg}$ body weight in $0.1 \mathrm{M}$ citrate buffer $(\mathrm{pH}=4.5)$. The rats were given $5 \%$ glucose solution from 4 to 8 hours to reduce STZ induced hypoglycemia (29), and fasting blood glucose was determined after $72 \mathrm{hrs}$ by glucometer. The rats with blood glucose level above $200 \mathrm{mg} / \mathrm{dL}$ were considered as diabetic and those with normal blood glucose level were re-injected STZ to induce T2DM.

\section{Preparation of Persea americana Fruit Juice and Dosage Calculation}

Persea americana mill fruit was purchased from Jimma town, $350 \mathrm{Km}$ South West of Addis Ababa, Ethiopia and identified by taxonomists in national herbarium, Addis Ababa University. The ripen fruit was washed carefully with distilled water and cut off by spoon, peeled off and the seed removed. Then the edible part of fruit was mixed by

blender to prepare a juice as consumed by humans. In our study, Persea americana juice was used to treat diabetic induced male albino Wistar rats. The juice was prepared by adding water to $80 \mathrm{gm}$ of wet fruit pulp up to $200 \mathrm{ml}$ mark. Then the juice was dried to know the weight of the fruit powder that is found in juice. The dry mass of the fruit in $200 \mathrm{ml}$ juice was found to be $44.5 \mathrm{gm}$. The volume of juice given to rats was also extrapolated from volume of human daily consumption, which is $(200 \mathrm{ml}$ of juice /day in $70 \mathrm{Kg})$. In this study the average body weight (BW) of rats were $250 \mathrm{gm}$. Therefore, the volume of juice given to rats was $0.71 \mathrm{ml}$. The approximate amount of Persea americana fruit pulp powder in $0.71 \mathrm{ml}$ of juice is approximately $158 \mathrm{mg}$. Accordingly, $158 \mathrm{mg}, 316 \mathrm{mg}$ and $474 \mathrm{mg}$ /250gm BW/day was considered as a low, moderate and high dose of Persea americana fruit pulp juice, respectively. Similarly, $0.7,1.4$ and $2.1 \mathrm{ml} / 250 \mathrm{gm} B W /$ day were considered as low, moderate and higher volume that is given to rats daily. The doses (158mg, 316mg and $474 \mathrm{mg} / 250 \mathrm{gm} \mathrm{BW/day)} \mathrm{were} \mathrm{converted} \mathrm{to} \mathrm{standard}$ unit $(\mathrm{mg} / \mathrm{Kg})$ as 632,1264 , and $1896 \mathrm{mg} / \mathrm{Kg} /$ day, respectively and used in this thesis document. The Persea Americana fruit juice was administered for six weeks orally from 9:30-10:30 AM.

\section{Extrapolation of Metformin Dose}

Safe and effective drug dosing is necessary regardless of its purpose of administration. There are several instances where in the initial dose of a particular drug is unavailable in a specific species. Therefore, choosing starting dose of such drugs for research, experiments, or clinical trials in animals and humans is a concern. The human dose of metformin drug was extrapolated to animal dose as follows (30). 
$\mathrm{AED} \mathrm{mg} / \mathrm{Kg}=$ Human dose in $\mathrm{mg} / \mathrm{Kg} \times \mathrm{Km}$ ratio where $\mathrm{Km}=\underline{\text { Human } \mathrm{Km}}$

Animal $\mathrm{Km}$

\section{Blood Sample Collection, Serum Preparation and Storage}

At the end of the experiment, the rats were fasted overnight and euthanized by anesthetizing with diethyl ether and then blood was collected by direct cardiac puncture to serum separator tube. Then, the blood was left at room temperature for 30 minutes to coagulate and centrifuged at $3000 \mathrm{rpm}$ for 10 minutes. The serum sample was transferred to necked tube and stored in deep freezer at $-80^{\circ} \mathrm{C}$ until the biochemical analyses were done.

\section{Biochemical Tests}

Total cholesterol (TC), triglyceride (TG), high density lipoprotein cholesterol (HDL-C), total protein (TP) and creatinine were determined with chemistry analyzer. Low density lipoprotein cholesterol (LDL-C) level was calculated using Frieldwald's formulae (31). Overnight fasting blood glucose was collected from the tail of the rats, and measured with One Touch GlucoSure on $0,14^{\text {th }}, 28^{\text {th }}$ and $42^{\text {th }}$ days of the experiment.

\section{Statistical Data Analysis}

The statistical data were analyzed using SPSS version 22. 00. The results of food consumption, body weight and biochemical parameters were expressed as mean \pm SEM. The statistical differences between the means were examined using analysis of variance (ANOVA) followed by Post Hoc Tukey's multiple comparisons. The p-values < 0.05 were declared as statistically significant.

\section{Results}

\section{Effect of Persea americana on Food Intake}

The food intake of diabetic control group rats was significantly increased $(p=0.001)$ when compared with normal control group. In high dose treated group, the mean pellet consumption was significantly reduced when compared with diabetic control group $(p=0.004)$. However, no significant difference was observed in moderate and high dose treated group compared to the normal control group (Table 1).

Table 1.

The effects of Persea americana mill fruit juice on food consumption

\begin{tabular}{|lllllllll|}
\hline Variable & Groups & & & & & & P- \\
& & & & & & & Value \\
& NC (n=6) & DC (n=6) & Mt (n=6) & $A(n=6)$ & $B(n=6)$ & $C(n=6)$ & \\
\hline \multirow{2}{*}{ FIT (gm/cag e/day) } & 135.88 & 149.03 & 143.45 & 145.60 & 142.29 & $137.19 \pm$ & \\
& \pm 4.117 & $\pm .458 \mathrm{a}$ & \pm 228 & \pm .494 & \pm 2.458 & $1.134^{\mathrm{b}}$ & $0.001^{*}$ \\
\hline
\end{tabular}

The values are expressed as mean \pm SEM. FIT- food intake; NC (I) - normal control; DC (II) - diabetic control; $M$-(III) metformin control; $A$ (IV) - low dose treated group; $B(V)$ - moderate dose treated group; $C$ (VI) - high dose treated 
group; * - significant difference between all groups as tested by one way ANOVA. The superscript letters "a" and " $b$ " indicate the significant differences compared to normal control and diabetic control groups, respectively.

\section{Effect of Persea americana on Body Weight}

Before the induction of T2DM, there was no significant difference in the mean values of body weight among all groups of the rats. However, at the end of experiment, the mean body weight of diabetic control, low dose Persea americana and metformin treated groups significantly decreased compared to body weight of normal control group ( $p=0.001 ; 0.007$ and 0.044$)$, respectively. Interestingly, the mean body weight of high dose treated group significantly increased compared with that of diabetic control group $(p=0.002)$. There was no significant difference in body weight between normal control and high dose treated groups (Figure 1).

\section{Effects of Persea americana on Fasting Serum Glucose Level}

Before treatment, the fasting serum glucose levels were significantly increased in all diabetic induced rats compared with normal control $(p<0.05)$. The mean value of fasting serum glucose was significantly reduced in rats treated with high dose of Persea americana on $4^{\text {th }}(p=0.036)$ and $6^{\text {th }}$ week $(p=0.012)$ compared with diabetic control group. Statistically significant decrease in serum glucose was also observed in moderate dose Persea americana juice treated group after the sixth week of treatment as compared with diabetic control group $(p=0.02)$. Metformin treated group showed significant reduction in fasting serum glucose on the $2^{\text {nd }}(p=0.044), 4^{\text {th }}(p=$ $0.021)$ and 6 th $(p=0.013)$ weeks of treatment, respectively compared with diabetic control group (Figure 2$)$.

\section{Effects of Persea americana on Lipid Profile}

After the induction of diabetes and subsequent treatment with Persea americana juice, the mean values of TC ( $p=$ $0.006)$ and LDL-C $(p=0.007)$ in high dose treated diabetic induced rats were significantly reduced when compared with the mean values in the diabetic control group. In metformin treated group, the mean values of $\operatorname{serum~TC~}(p=$ $0.003)$ and LDL-C $(p=0.006)$ were significantly decreased compared with that of the diabetic control group. The mean values of TC $(p=0.002)$ and LDL-C $(p=0.004)$ in diabetic control group were significantly increased compared with that of the normal control group. Interestingly, there was no significant difference in mean values of TC and LDL-C in moderate and high dose Persea americana juice, and metformin treated groups when compared to normal control group. The mean value of fasting serum HDL-C was decreased in diabetic control rats, compared to normal control group $(p=0.002)$. On the other hand, the mean value of HDL-C was significantly increased in moderate $(p=0.046)$ and high $(p=0.034)$ doses of Persea americana and metformin $(p=0.014)$ treated groups as compared with diabetic control group. No significant difference was found in mean HDL-C level among the normal control, metformin control and high dose treated groups. The mean value of TG did not show any significant difference among all the study groups $(p>0.05)$, (Table 2$)$. 
Table 2.

Effects of Persea americana fruit juice on lipid profile in diabetic rats

\begin{tabular}{|c|c|c|c|c|c|c|c|c|}
\hline \multirow[b]{2}{*}{ Variable } & \multirow[b]{2}{*}{$\begin{array}{l}\mathrm{NC} \\
(\mathrm{n}=6)\end{array}$} & \multirow[b]{2}{*}{$D C(n=6)$} & \multirow[b]{2}{*}{$M t(n=6)$} & \multicolumn{3}{|l|}{ Group } & \multicolumn{2}{|c|}{ ANOVA } \\
\hline & & & & $A(n=6)$ & $B(n=6)$ & $C(n=6)$ & $\mathrm{F}$ & $\begin{array}{l}p- \\
\text { value }\end{array}$ \\
\hline \multirow{2}{*}{$\begin{array}{l}\text { TG } \\
\text { (mg/dL) }\end{array}$} & 114.2 & 185.8 & 117.8 & $151 \pm 13.6$ & 146.2 & 139.8 & 1.247 & 0.312 \\
\hline & \pm 5.5 & \pm 49.7 & \pm 20.9 & & \pm 5.6 & \pm 10.1 & & \\
\hline \multirow{2}{*}{$\begin{array}{l}\mathrm{TC} \\
(\mathrm{mg} / \mathrm{dL})\end{array}$} & 109.7 & $211.7^{a}$ & $135.2^{b}$ & 187.8 & 158.8 & $141 \pm 7.2^{b}$ & 4.7 & $0.006 *$ \\
\hline & \pm 4.3 & \pm 13.1 & \pm 11.2 & \pm 21.4 & \pm 12.8 & & & \\
\hline \multicolumn{9}{|l|}{ HDL-C } \\
\hline$(\mathrm{mg} / \mathrm{dL})$ & $41.2 \pm 0.8$ & $26.3 \pm 0.6^{\mathrm{a}}$ & $40.7 \pm 0.8^{b}$ & $28.7 \pm 0.7$ & $34.2 \pm 0.6^{\mathrm{b}}$ & $38.8 \pm 0.6^{b}$ & 4.3 & $0.007^{*}$ \\
\hline $\begin{array}{l}\text { LDL-C } \\
\text { (mg/dL) }\end{array}$ & $50.6 \pm 4.1$ & $151.4 \pm 13.1^{\mathrm{a}}$ & $77.2 \pm 10.4^{b}$ & $126 \pm 23.4$ & $\begin{array}{c}95.4 \\
\pm 30.5\end{array}$ & $77.6 \pm 9.5^{b}$ & 4.7 & $0.004^{*}$ \\
\hline
\end{tabular}

The results are expressed as mean \pm SEM ( $n=6)$; NC- normal control; $D C$ - diabetic control; $M t$ - metformin control; $A$ - low dose treated group; $B$ - moderate dose treated group; C - high dose treated group; TG - Triglyceride; TC-Total Cholesterol; HDL-C, High Density Lipoprotein Cholesterol; LDL-C, Low Density Lipoprotein Cholesterol; a,b statistically significant $(p<0.05)$ compared with normal and diabetic control, respectively.

\section{Effects of Persea americana on Total Protein and Creatinine}

Regarding serum total protein and creatinine levels, no significant difference was observed among the study groups (Table 3).

Table 3.The effects of Persea americana fruit juice on total protein and creatinine of diabetic rats

\begin{tabular}{|lllllllll|} 
variables & $\mathrm{NC}(\mathrm{n}=6)$ & $\mathrm{DC}(\mathrm{n}=6)$ & $\mathrm{Mt}(\mathrm{n}=6)$ & $\mathrm{A}(\mathrm{n}=6)$ & $\mathrm{B}(\mathrm{n}=6)$ & $\mathrm{C}(\mathrm{n}=6)$ & $\mathrm{F}$ & $\mathrm{p}$-value \\
\hline $\mathbf{T P}(\mathbf{m g} / \mathbf{d L})$ & $5.32 \pm .34$ & $3.7 \pm .23$ & $5.23 \pm .65$ & $4.37 \pm .46$ & $4.92 \pm .5$ & $5.05 \pm .54$ & 1.74 & 0.157 \\
& & & & & & & & \\
\hline $\begin{array}{l}\text { Creatinine } \\
\text { (mg/dL) }\end{array}$ & $0.53 \pm .06$ & $0.90 \pm .13$ & $0.54 \pm .09$ & $0.61 \pm .07$ & $0.60 \pm .08$ & $0.59 \pm .09$ & 2.49 & 0.053 \\
\hline
\end{tabular}

The results are expressed as mean \pm SEM $(n=6)$; TP - Total Protein; NC - normal control; DC - diabetic control; Mt metformin control; $A$ - low dose treated group; $B$ - moderate dose treated group; $C$ - high dose treated group

\section{Discussion}

Persea americana is a fruit used as human food and in treating different diseases. In the present study, Persea americana juice was used to treat high fat diet and low dose STZ induced T2DM in male albino Wistar rats for six 
weeks. The effect of Persea americana on food consumption, body weight, fasting blood glucose, lipid profile, total protein and creatinine was investigated in the diabetic rats.

In this study, food intake was higher in diabetic control group compared with in normal control group. It was also observed that the food consumption in high dose and moderate dose Persea americana juice treated groups of rats was lower than in diabetic control group. In addition, the pellet intake was reduced in the high dose treated group when compared with pellet intake in metformin treated group. The appetite decreasing of effect of Persea americana fruit juice is due to the presence of dietary fiber and fruit oil matrix, which acts by slowing gastric emptying and increasing early satiety in the rats $(32,33)$.

Slight body weight loss was observed in HFD and low dose STZ-induced diabetic rats following oral administration of both Persea americana fruit juice and metformin. In diabetic rats, decreased body weight may be due to an excessive breakdown of tissue proteins and lipid to provide energy as the body cannot use carbohydrate due to insulin insufficiency (34). The improvement in body weight seen in diabetic rats treated with Persea americana juice is most probably due to an improved metabolic activity that makes the body system more capable of maintaining blood glucose homeostasis (35). Similarly, $7 \mathrm{mg} / \mathrm{Kg}$ of metformin treated rats gained body weight when compared with the diabetic group after six weeks of the treatment. However, the effect of metformin was less compared with that of Persea americana juice in normalizing body weight. The study done by Duerta et al. also reported that the $\beta$ - sitosterol found in Persea americana showed body weight loss activity by reducing compulsive eating and fat accumulation in the abdominal region (36). The bioactive compounds found in Persea americana fruit juice may help in suppressing free radicals generation due to hyperglycemia and control muscle over wasting that resulted from glycemic control in treated diabetic rats, and ultimately lead to normalize the level of body weight (37).

In the first week of the experiment, fasting blood glucose concentration was elevated in all diabetic induced rats compared with normal control rats. This increase in fasting blood glucose concentration is an important characteristic feature of T2DM. However, for diabetic rats that received Persea americana juice, their fasting blood glucose was non-significantly reduced in the second week of the experiment. The fasting blood glucose level was significantly decreased on fourth and sixth weeks of the experiment in rats treated with moderate and high dose of Persea americana pulp juice when compared with glucose level of diabetic control rats. These results agree with Rao and Adinew's results that reported albino Wistar rats received ethanolic extract of Persea americana fruit 300 $\mathrm{mg} / \mathrm{Kg} /$ day, orally for 4 weeks, showed a decrease in the fasting blood glucose level, glycosylated hemoglobin, blood urea, and serum creatinine (35). Similar finding was also reported by Thenmozhi et al. in which treatment of Sprague Dawley rats with n-hexane fraction from hydromethanolic (2:3) extract of Persea americana fruit $30 \mathrm{mg} / \mathrm{Kg}$ orally for 8 weeks showed decrease in fasting blood glucose level (38). A randomized clinical trials study done by Sabate et al. on 26 healthy overweight individuals also revealed that consumption of half avocado significantly reduced the blood insulin and glucagon-like peptide-1 levels (39). Moreover, the results of Wien et al. investigation on healthy overweight adults showed that avocado in lunch meal attenuated rise in postprandial blood insulin levels $30 \mathrm{~min}$ after start of the lunch meal and diminished the desire to eat compared with the avocado-free control group (33). On the other hand, low dose Persea americana juice did not cause significant change in fasting blood glucose throughout six weeks of the treatment, but decreased the levels to some extent.

The antihyperglycemic effect of avocado fruits may be related to insulin mimetic or stimulatory effect and its ability to stimulate the remaining pancreatic $\beta$-cells in animal models, making them able to secrete more insulin (35). The phytochemicals like flavonoids, saponins, tannins and alkaloids found in avocado act as anti-oxidants 
and contain insulin stimulating substances such as insulin receptor substrate, glycogen synthase and glucose dependent insulotropic polypeptide. Persea americana could decrease glucose level by increasing insulin secretion or peripheral glucose utilization in the gut of normal treated rats (40). The D - manno heptulose found in Persea americana may also be responsible for hypoglycemic effect by decreasing the rate of glycolysis via hexokinase inhibition and weight control via appetite reduction.

In metformin treated group, the fasting blood glucose is significantly reduced starting from second week of the experiment when compared with diabetic control group. Metformin reduces hyperglycemia by reducing gluconeogenesis, increasing insulin receptor sensitivity, especially in muscle cells, and decreasing glucose uptake in the intestine (41-43). On the sixth week, no significant difference was found in fasting glucose levels among rats treated with moderate and high dose of Persea americana juice and metformin.

In addition to its anti-hyperglycemic effect, Persea americana fruit juice also showed the ability to alter lipid profile (TC, LDL-C -C, TG, and HDL-C -C) levels in diabetic albino Wistar rats. The abnormally high concentration of serum lipids in diabetes is mainly due to the increase in the mobilization of free fatty acids from the peripheral depots as insulin inhibits the hormone sensitive lipase. In our study, there was a significant increase in the serum TC and LDL-C levels in diabetic rats as compared with normal control rats. The elevated TG level in diabetic rats might be due to the consequence of increased synthesis of triglyceride rich lipoprotein particles (VLDL-C) in the liver and diminished catabolism. Since insulin has a potent inhibitory effect on lipolysis in the adipocytes, insulin deficiency is associated with excess lipolysis and increased influx of free fatty acids to the liver (44). The increase in LDL-C and VLDL-C levels in the HFD/low dose STZ induced diabetic rats might also be due to over production of LDL-C and VLDL-C by the liver through stimulation of hepatic triglyceride synthesis as a result of free fatty acid influx. The increased pool of triacylglycerol-rich lipoproteins, mainly VLDL-C1 observed in type 2 diabetes promotes CETPmediated triacylglycerol enrichment of HDL-C particles, and as a consequence enhances HDL-C catabolism, while depleting them from cholestryl esters, thus decreasing HDL-C cholesterol level (45).

Administration of high dose of Persea americana fruit juice decreased the mean serum TC and LDL-C levels in HFD and low STZ induced diabetic rats compared with diabetic control group. Our results agree with of PahuaRamos et al.'s results in which administration of reduced-calorie avocado paste $2 \mathrm{~g} / \mathrm{Kg} /$ day for 7 weeks showed a significant reduction in TC, LDL-C-C and increased insulin sensitivity in high cholesterol and fructose diet fed rats (46). Similar in a study done by Elbadrawy and Shelbaya, administration of hydro-alcoholic extract of avocado130 and $150 \mathrm{mg} / \mathrm{Kg} /$ day administrated via stomach tube for 8 weeks showed a significant reduction in serum cholesterol, LDL-C-C, and VLDL-C-C, while, the serum level of HDL-C-C was enhanced in HCD rats (47). In addition, in study done by Al-Dosari, oral administration of Persea americana pulp 1 and $2 \mathrm{ml} /$ rat/day for 10 weeks showed a significant decrease in serum cholesterol, LDL-C-C, VLDL-C-C and TG levels (48). The LDL-C and TC reducing effect of Persea americana fruit pulp juice is due to its phytosterols, phytostanols and dietary fiber, which help reduce cholesterol reabsorption in the intestine and promoting fecal cholesterol excretion, which in turn reduces the level of LDL-C in plasma, increasing the amount of cholesterol excreted from the body and decrease in hepatic cholesterol synthesis $(49,50)$.

In addition, the soluble dietary fiber found in avocado juice pulp may decrease the LDL-C and TC levels by binding to bile acid and alters micelle formation, and results in decreased micelle absorption in the small intestine. Moreover, the extract of avocado fruit inhibits the action of acetyl-CoA carboxylase, a key enzyme catalyzing the committed in fatty acid synthesis (51). Treating diabetic rats with metformin decreased the serum TC and LDL-C at 
the end of the experiment ( $6^{\text {th }}$ weeks). Metformin increases insulin sensitivity by decreasing the rate of lipolysis there by slowing the conversion of FFA to lipoprotein precursors in the liver.

Interestingly, the mean value of HDL-C was significantly increased in in diabetic rats treated with double and triple doses of Perseaamericana fruit juice when compared with diabetic control rats. This result is in line with the result of Elbadrawy and Shelbaya, in which administration of hydroalcolic extract of avocado pulp $150 \mathrm{mg} / \mathrm{Kg}$ for 8 weeks increased HDL-C in HCD rats (47). The possible mechanism by which avocado enhances HDL-C serum level may be by regulating the hydrolysis of certain lipoproteins and their selective uptake, and metabolism by different tissues due to presence of tocopherols, phytosterols and polyphenols in the fruit. Avocado also modifies the structure of the HDL-C lipoprotein by increasing paraoxonase-1 enzyme activity, which is responsible for the hydrolysis of lipid hydroperoxides (52). In the same way, the HDL-C cholesterol level was significantly increased in diabetic rats treated with metformin drug compared with in diabetic control rats.

On the other hand, no dose of avocado showed significant effect on serum TG level in all treatment groups compared to diabetic control group $(p>0.05)$. However, there was a decrease in mean value of serum TG after the treatment. This result is in agreement with Pieterse's, in which $200 \mathrm{~g} /$ day of avocado substituted for $30 \mathrm{~g}$ of other mixed dietary fats for 6 weeks didn't significantly change the TG, HDL-C, LDL-C and TC levels in energy-restricteddiet volunteers (53). On the contrary to our study, study done by Shehata and Soltan revealed treatment of hypercholesteromic rat with $30 \%$ avocado fruit for 4 -weeks reduced TG levels significantly (54). This difference might be due to the difference in extraction method used.

In T2DM, insulin resistance does not alter only glucose and lipid metabolism, but also protein metabolism. In the present study, the total protein level was reduced in diabetic rats relative to in normal control rats. Diabetes mellitus reduces total protein level by increasing muscle proteolysis, reducing protein synthesis and stimulating hepatic gluconeogenesis. Although the differences were not significant, treating diabetic rats with different doses of avocado fruit juice was found to improve the total protein concentration. The metformin treated group also did not show significant difference in protein level compared to diabetic control group. In diabetic rats, breaking down of liver and plasma protein enhances serum creatinine level. The administration of Persea americana fruit juice and metformin to diabetic rats decreased serum creatinine level insignificantly. This indicates that the effect of Persea americana in normalizing protein metabolism disturbance is comparable to that of metformin.

\section{Conclusion}

The present study revealed that the antihyperglycemic and antihperlipidemic effect of Persea americana fruit juice in HFD/low dose STZ induced T2DM albino Wistar rats is a dose dependent. Particularly, high dose (1896 $\mathrm{mg} / \mathrm{Kg} /$ day) of Persea americana juice significantly reduced pellet consumption than metformin. Similarly, Persea americana friut juice showed the effect of normalizing body weight and reducing fasting blood glucose level. Treating diabetic rats with Persea americana juice $(1264 \mathrm{mg} / \mathrm{Kg}, 1896 \mathrm{mg} / \mathrm{Kg} /$ day) showed significant fasting blood glucose reduction on the $4^{\text {th }}$ and $6^{\text {th }}$ weeks. It was also found that Persea americana fruit pulp also has a potential reducing effect on LDL-C, TC and increasing effect on HDL-C in STZ and HFD induced diabetic albino rats. Overall, the findings of our study showed that Persea americana fruit pulp juice has the effect of normalizing metabolic disturbances of carbohydrate and lipid caused by T2DM and HFD, hence it may be helpful in reducing the risk of diabetic complications. 


\section{List Of Abbreviations And Acronyms}

\begin{tabular}{|ll|}
\hline AED & Animal Equivalent Dose \\
\hline ANOVA & Analysis of Varience \\
\hline DM & Diabetes Mellitus \\
\hline FBG & Fasting Blood Glucose \\
\hline HDL-C-C & High Density Lipoprotein Cholesterol \\
\hline HFD & High Fat Diet \\
\hline LDL-C & Low Density Lipoprotien Cholesterol \\
\hline STZ & Streptozotocin \\
\hline T1DM & Type 1 Diabetes Mellitus \\
\hline T2DM & Type 2 Diabetes Mellitus \\
\hline TC & Total Cholesterol \\
\hline TG & Triglyceride \\
\hline Rpm & Revolution per Minute \\
\hline WHO & World Health Organization \\
\hline
\end{tabular}

\section{Declarations}

\section{Acknowledgment}

We would like to express our earnest gratitude to Dilla University and Addis Ababa University for funding this research project. We are extremely grateful to the Department of Medical Biochemistry, Addis Ababa University, for its indispensable support and coordination in smooth running of this project.

\section{Authors' contributions}

TG conceived and designed the study, conducted the experiment, analyzed and interpreted the data, and wrote the manuscript. SG and AT designed the study, analyzed and interpreted the data, and TOF assisted in study designing, conducting the experiment, data analysis and interpretation, and manuscript writing. All the authors read, commented on, and contributed to the submitted and revised manuscript.

\section{Funding}

The research was funded by Dilla University and Addis Ababa University. The funders had no role in designing the study, collection, analysis, and interpretation of data as well as in the writing of the manuscript.

\section{Availability of data and materials}

All necessary data and materials are included in this manuscript and supplementary data and materials related to the article can be obtained from the authors when necessary. 


\section{Competing interests}

The authors declare that they have no competing interests.

\section{References}

1. Marrero-Faz E, Sanchez-Calero J, Young L, Harvey A. Inhibitory effect of Persea americana Mill leaf aqueous extract and its fractions on PTP1B as therapeutic target for type 2 diabetes. Boletín Latinoamericano y del Caribe de Plantas Medicinales y Aromáticas. 2014;13(2):144-51.

2. Ighodaro 0 , Omole J, Adejuwon A, Odunaiya A. Effects of Parinari polyandra seed extract on blood glucose level and biochemical indices in Wistar Rats. International Journal of Diabetes Research. 2012;1(4):68-72.

3. Ambachew Y, Kahsay S, Tesfay R, Tesfahun L, Amare H, Gebreegzihabiher G. Prevalence of diabetes mellitus among patients visiting medical outpatient department of Ayder Referral Hospital, Mekelle, Ethiopia: A three years pooled data. Int J Pharma Sci Res. 2015;6(02):435-9.

4. Cho N, Shaw J, Karuranga S, Huang Y, da Rocha Fernandes J, Ohlrogge A, et al. IDF Diabetes Atlas: Global estimates of diabetes prevalence for 2017 and projections for 2045. Diabetes research and clinical practice. 2018;138:271-81.

5. Kamagate M, Koffi E, Kadja AB, Camille K, Balayssac E, Daubrey-Potey T, et al. Acute toxicity and hypoglycaemic activity of the leaf extracts of Persea americana Mill.(Lauraceae) in Wistar rats. African Journal of Pharmacy and Pharmacology. 2016;10(33):690-8.

6. Yigazu DM, Desse TA. Glycemic control and associated factors among type 2 diabetic patients at Shanan Gibe Hospital, Southwest Ethiopia. BMC research notes. 2017;10(1):1-6.

7. Association AD. Diagnosis and classification of diabetes mellitus. Diabetes care. 2014;37(Supplement 1):S81S90.

8. Edem D, Ekanem I, Ebong P. Effect of aqueous extracts of alligator pear seed (Persea americana Mill) on blood glucose and histopathology of pancreas in alloxan-induced diabetic rats. Pakistan Journal of Pharmaceutical Sciences. 2009;22(3).

9. Tama BA. An early detection method of type-2 diabetes mellitus in public hospital. Telkomnika. 2011;9(2):28794.

10. Alhassan A, Sule M, Atiku M, Wudil A, Abubakar H, Mohammed S. Effects of aqueous avocado pear (Persea americana) seed extract on alloxan induced diabetes rats. Greener Journal of Medical Sciences. 2012;2(1):005-11.

11. Del Toro-Equihua M, Velasco-Rodríguez R, López-Ascencio R, Vásquez C. Effect of an avocado oil-enhanced diet (Persea americana) on sucrose-induced insulin resistance in Wistar rats. journal of food and drug analysis. 2016;24(2):350-7.

12. Park C, Cuypers L, Sin A. Impact of avocado enriched diets on serum lipids of diabetic patients. J Cardiovasc Dis. 2013;1:13-4.

13. Rao UM, Haque M, Baig AA. Insulin stimulative and anti-oxidative effects of Persea americana fruit extract on streptozotocin induced hyperglycemic rats. Insulin. 2011;4(1).

14. Arokiyaraj S, Balamurugan R, Augustian P. Antihyperglycemic effect of Hypericum perforatum ethyl acetate extract on streptozotocin-induced diabetic rats. Asian Pacific journal of tropical biomedicine. 2011;1(5):38690. 
15. Dusane MB, Joshi BN. Beneficial effect of flax seeds in streptozotocin (STZ) induced diabetic mice: isolation of active fraction having islet regenerative and glucosidase inhibitory properties. Canadian journal of physiology and pharmacology. 2013;91(5):325-31.

16. Naveh E, Werman MJ, Sabo E, Neeman I. Defatted avocado pulp reduces body weight and total hepatic fat but increases plasma cholesterol in male rats fed diets with cholesterol. The Journal of nutrition. 2002;132(7):2015-8.

17. Monika P, Geetha A. Effect of hydroalcoholic fruit extract of Persea americana Mill. on high fat diet induced obesity: a dose response study in rats. Indian J Exp Biol. 2016;54(6):370-8.

18. Mahmoed MY, Rezq AA. Hepatoprotective effect of avocado fruits against carbon tetrachloride-induced liver damage in male rats. World Applied Sciences Journal. 2013;21(10):1445-52.

19. Christiansen BA, Bhatti S, Goudarzi R, Emami S. Management of osteoarthritis with avocado/soybean unsaponifiables. Cartilage. 2015;6(1):30-44.

20. Ranade SS, Thiagarajan P. A review on Persea americana Mill.(avocado)-its fruits and oil. Int J PharmTech Res. 2015;8(6):72-7.

21. Paul R, Kulkarni P, Ganesh N. Avocado fruit (Persea americana Mill) exhibits chemo-protective potentiality against cyclophosphamide induced genotoxicity in human lymphocyte culture. J Exp Ther Oncol. 2011;9(3):221-30.

22. Soong Y-Y, Barlow PJ. Antioxidant activity and phenolic content of selected fruit seeds. Food chemistry. 2004;88(3):411-7.

23. Imafidon K, Amaechina F. Effects of aqueous seed extract of Persea americana Mill.(avocado) on blood pressure and lipid profile in hypertensive rats. Adv Biol Res. 2010;4(2):116-21.

24. Arifin WN, Zahiruddin WM. Sample size calculation in animal studies using resource equation approach. The Malaysian journal of medical sciences: MJMS. 2017;24(5):101.

25. Lelyana R. Effect of coffee daily consumption on uric acid level and body weight to Prevent metabolic syndrome. J Nanomed Nanotechnol. 2016;7(5):1-5.

26. Louwe MC, van der Hoorn JW, van den Berg SA, Jukema JW, Romijn JA, Willems van Dijk K, et al. Genderdependent effects of high-fat lard diet on cardiac function in C57BI/6J mice. Applied physiology, nutrition, and metabolism. 2012;37(2):214-24.

27. Srinivasan K, Viswanad B, Asrat L, Kaul C, Ramarao P. Combination of high-fat diet-fed and low-dose streptozotocin-treated rat: a model for type 2 diabetes and pharmacological screening. Pharmacological research. 2005;52(4):313-20.

28. Skovs $\varnothing$ S. Modeling type 2 diabetes in rats using high fat diet and streptozotocin. Journal of diabetes investigation. 2014;5(4):349-58.

29. Krishnan K, Vijayalekshmi N, Helen A. Methanolic extract of Costus igneus (NE Br) alleviates dyslipidemia in diabetic rats. Asian J Pharm Clin Res. 2011;4:154-7.

30. Nair AB, Jacob S. A simple practice guide for dose conversion between animals and human. Journal of basic and clinical pharmacy. 2016;7(2):27.

31. Dansethakul P, Thapanathamchai L, Saichanma S, Worachartcheewan A, Pidetcha P. Determining a new formula for calculating low-density lipoprotein cholesterol: data mining approach. EXCLI journal. 2015;14:478.

32. Naveh E, Werman MJ, Sabo E, Neeman I. Defatted avocado pulp reduces body weight and total hepatic fat but increases plasma cholesterol in male rats fed diets with cholesterol. The Journal of nutrition. 
2002;132(7):2015-8.

33. Wien M, Haddad E, Oda K, Sabaté J. A randomized 3x3 crossover study to evaluate the effect of Hass avocado intake on post-ingestive satiety, glucose and insulin levels, and subsequent energy intake in overweight adults. Nutrition journal. 2013;12(1):1-9.

34. Gouegni E, Abubakar H. Phytochemical, toxicological, biochemical and haematological studies on avocado (Persea americana) in experimental animals. Nigerian Food Journal. 2013;31(1):64-9.

35. Rao UM, Adinew B. Remnant B-cell-stimulative and anti-oxidant effects of Persea americana fruit extract studied in rats introduced into streptzotocin-induced hyperglycaemic state. African Journal of Traditional, Complementary and Alternative Medicines. 2011;8(3).

36. Duarte PF, Chaves MA, Borges CD, Mendonça CRB. Avocado: characteristics, health benefits and uses. Ciência Rural. 2016;46(4):747-54.

37. Auddy B, Ferreira M, Blasina F, Lafon L, Arredondo F, Dajas F, et al. Screening of antioxidant activity of three Indian medicinal plants, traditionally used for the management of neurodegenerative diseases. Journal of ethnopharmacology. 2003;84(2-3):131-8.

38. Thenmozhi A, Shanmugasundaram C, Rao UM. Biochemical evaluation of anti-diabetic phytomolecule through bioactivity guided solvent fractionation and subfractionation from hydromethanolic (2: 3 ) extract of Alligator pear Fruit in streptozotocin induced diabetic rats. Journal of Applied Pharmaceutical Science. 2012;2(1):61.

39. Sabaté J, Wien M, Haddad E. Post-ingestive effects of avocados in meals on satiety and gastric hormone blood levels. Human Health Nut. 2015:459-61.

40. Aigbiremolen A, Ativie R, Aisuodionoe M, Odigie O, Igweh J, Egwaoje M. Effect of Aqueous Extract of Persea americana Seed on Blood Glucose in Alloxan-induced Diabetic Wistar Rats. 9 (3), 1-10. 2018.

41. Derosa G, Maffioli P. Thiazolidinediones plus metformin association on body weight in patients with type 2 diabetes. Diabetes Research and Clinical Practice. 2011;91(3):265-70.

42. Harvey RA, Clark M, Finkel R, Rey J, Whalen K. Lippincott's illustrated reviews: Pharmacology: Philadelphia; 2012.

43. Arbeláez-Quintero I, Palacios M. To use or not to use metformin in cerebral ischemia: a review of the application of metformin in stroke rodents. Stroke research and treatment. 2017;2017.

44. Bays HE, Toth PP, Kris-Etherton PM, Abate N, Aronne LJ, Brown WV, et al. Obesity, adiposity, and dyslipidemia: a consensus statement from the National Lipid Association. Journal of clinical lipidology. 2013;7(4):304-83.

45. Vergès B. Pathophysiology of diabetic dyslipidaemia: where are we? Diabetologia. 2015;58(5):886-99.

46. Pahua-Ramos ME, Garduño-Siciliano L, Dorantes-Alvarez L, Chamorro-Cevallos G, Herrera-Martínez J, OsorioEsquivel 0 , et al. Reduced-calorie avocado paste attenuates metabolic factors associated with a hypercholesterolemic-high fructose diet in rats. Plant foods for human nutrition. 2014;69(1):18-24.

47. Elbadrawy E, Shelbaya L. Hypolipidemic activities of hydroalcoholic extract of avocado fruit on high cholesterol fed diet in rats and its antioxidant effect in vitro. J Am Sci. 2013;9:337-43.

48. Al-Dosari MS. Hypolipidemic and antioxidant activities of avocado fruit pulp on high cholesterol fed diet in rats. African Journal of Pharmacy and Pharmacology. 2011;5(12):1475-83.

49. Ras RT, Geleijnse JM, Trautwein EA. LDL-cholesterol-lowering effect of plant sterols and stanols across different dose ranges: a meta-analysis of randomised controlled studies. British Journal of Nutrition. 2014;112(2):214-9. 
50. Wang DQ-H. Regulation of intestinal cholesterol absorption. Annu Rev Physiol. 2007;69:221-48.

51. Hashimura H, Ueda C, Kawabata J, Kasai T. Acetyl-CoA carboxylase inhibitors from avocado (Persea americana Mill) fruits. Bioscience, biotechnology, and biochemistry. 2001;65(7):1656-8.

52. Boshtam M, Emami Razavi A, Pourfarzam M, Ani M, Naderi GA, Basati G, et al. Serum paraoxonase 1 activity is associated with fatty acid composition of high density lipoprotein. Disease markers. 2013;35.

53. Pieterse Z, Jerling J, Oosthuizen W, Kruger H, Hanekom S, Smuts C, et al. Substitution of high monounsaturated fatty acid avocado for mixed dietary fats during an energy-restricted diet: effects on weight loss, serum lipids, fibrinogen, and vascular function. Nutrition. 2005;21(1):67-75.

54. Shehata MM, Soltan SS. Effects of bioactive component of kiwi fruit and avocado (fruit and seed) on hypercholesterolemic rats. World Journal of Dairy \& Food Sciences. 2013;8(1):82-93.

\section{Figures}

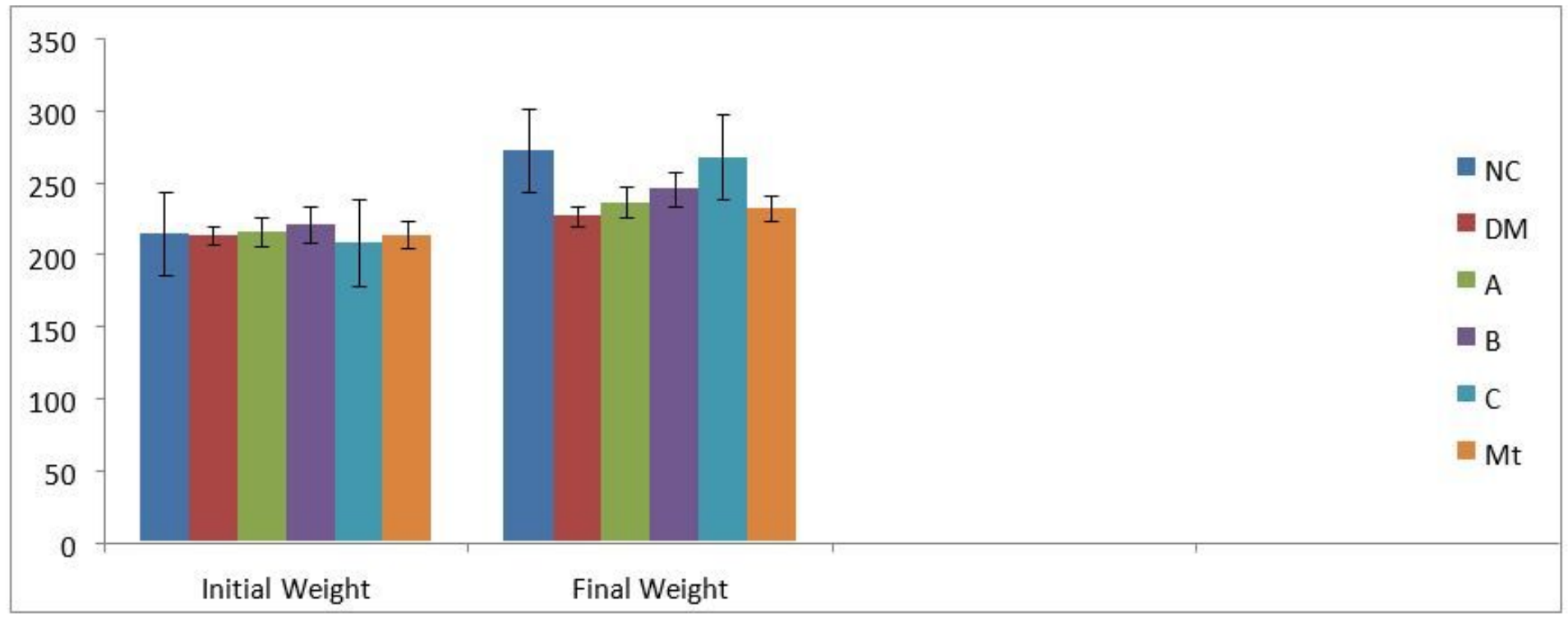

\section{Figure 1}

The effects of Persea americana mill pulp juice on body weight in HFD and low dose STZ induced diabetic albino Wistar rats. The results are expressed as mean \pm SEM ( $n=6)$; NC - normal control; DC - diabetic control; Mt metformin control; A - low dose, B - moderate dose and C - high dose treated groups, respectively. 


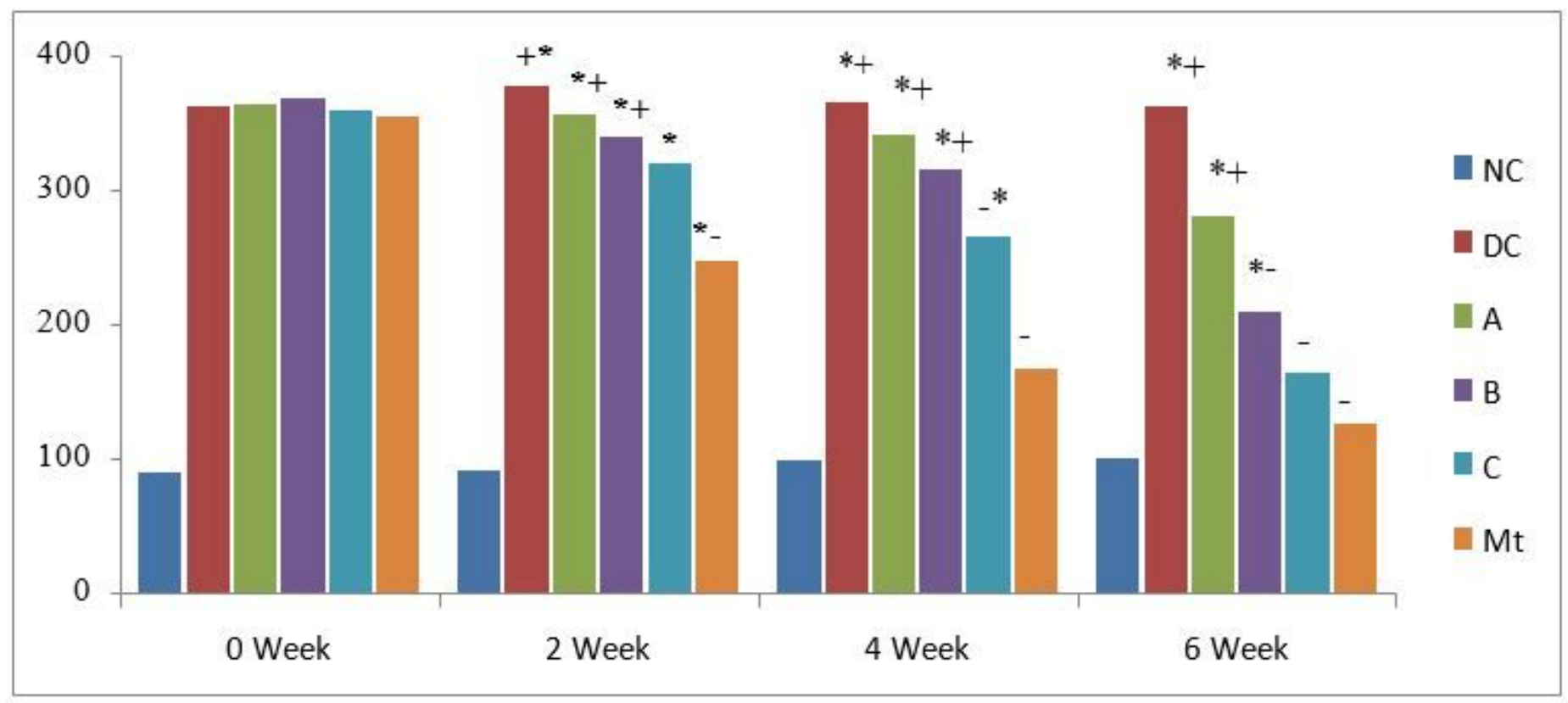

Figure 2

The effects of Persea americana mill on fasting serum glucose of HFD \&low dose STZ induced diabetic albino Wistar rats. The results are described as mean \pm SEM, $n=6$; NC- normal control; DC-diabetic control; Mt-metformin control; A-low dose treated group; B -moderate dose treated group; C -high dose treated group. The symbols “*”, "-” and "+" indicate significant differences when compared with normal, diabetic and metformin control groups, respectively. 